
\title{
$\begin{array}{ll}\text { Research Square } & \begin{array}{l}\text { Preprints are preliminary reports that have not undergone peer review. } \\ \text { They should not be considered conclusive, used to inform clinical practice, } \\ \text { or referenced by the media as validated information. }\end{array}\end{array}$
}

\section{Online Positive Parenting Program for Promoting Parenting Competencies and Skills: Randomised Controlled Trial}

\author{
Sararat Tuntipuchitanon \\ Chulalongkorn University \\ Ing-on Kangwanthiti \\ Chulalongkorn University \\ Ketsupar Jirakran \\ Chulalongkorn University \\ Pon Trairatvorakul ( $\square$ pon.t@chula.md) \\ Chulalongkorn University \\ Weerasak Chonchaiya \\ Chulalongkorn University
}

\section{Research Article}

Keywords: Promoting population quality, Online Positive Parenting Program, Promoting Parenting Competencies, Skills, Randomised Controlled Trial

Posted Date: October 28th, 2021

DOI: https://doi.org/10.21203/rs.3.rs-967394/v1

License: (0) (i) This work is licensed under a Creative Commons Attribution 4.0 International License. Read Full License 


\section{Abstract}

Background: Parenting programs, albeit effective, are not readily accessible to the general public, particularly during the COVID-19 pandemic.

Methods: In 103 healthy caregiver-child dyads, we investigated the effectiveness of online positive parenting program on parenting sense of competencies (primary outcome), parenting styles and behavioural concerns of children aged 3-6 years (secondary outcomes) between 2 blinded, parallel groups. After block of 4 randomisations, intervention group $(n=52)$ attended 8-session, intensive video conference while active control group $(n=51)$ received weekly general education via communication application. Outcomes were measured at baseline, 8 and 14 weeks.

Results: Most parents from both groups had high education and household income. At 14 weeks, the intervention group reported higher sense of competence (Wald 9.63, $p=0.008$ ); both groups reported using more authoritative parenting style (Wald 15.52, $p \leq 0.001)$ from Generalised Estimating Equations model. Compared to baseline, both groups had significant reduction of children's emotional problems at 14 weeks (mean change: Intervention $=-0.44, p=0.033$; Control $=-0.30, p=0.046$ ) and behavioural problems over time (Wald 7.07, $p=0.029$ ).

Conclusion: Online positive parenting program offered an easily accessible, primary preventive measure to mitigate behavioural concerns and improve parental competency.

Clinical Trial Registration: Thai Clinical Trials Registry; https://www.thaiclinicaltrials.org/; TCTR20201030001; October 30, 2020

\section{Introduction}

Promoting population quality is paramount and associated with optimal physical and mental health ${ }^{1}$. A meta-analysis of the prevalence of mental disorders in children and adolescents from 1985-2012 by Polanczyk and team found that disruptive behavioural problems were reported in approximately $5.7 \%^{2}$. Population-based cohort studies have observed that childhood disruptive behavioural disorders are precursors to a wide range of negative outcomes, including peer rejection, school failure, psychopathologic conditions, suicidality and criminality ${ }^{3-12}$. Children's behavioural problems also affect parental depression and stress $^{13,14}$. In addition to social, environmental, and economic pressure, parental stress may be influenced by parents' own physical and mental health, child's temperament and response to parenting ${ }^{15}$.

Positive parenting skills are built on the continuous development of parent's self-efficacy, the ability to set appropriate environments and expectations for children and manage difficult situations, and the capability to identify accurate and relevant parenting knowledge or assistance ${ }^{16,17}$. Parents with positive parenting skills can help their children to adapt and respond to stress appropriately ${ }^{18}$. Another important parental factor, parental competencies, can help improve parenting capacities ${ }^{17,19}$. Moreover, positive parent's emotion can reduce behavioural and physical health problems in children and strengthen the parent-child relationship ${ }^{16,20-22}$.

Positive parenting programs include providing parental assistance and knowledge, introducing reliable and helpful resources, enhancing parenting skills and providing economic and social support. Besides the popular Triple P-Positive Parenting Program aimed at preventing behavioural and emotional problems in children and adolescents ${ }^{22,23}$, other positive parenting programs were effective in reducing parents' mental health problems and children's behavioural and emotional problems, especially disruptive behavioural disorders ${ }^{22,24-28}$. Moreover, parent training programs are associated with better child's social, emotional and behavioural outcomes which in turn improve parent-child relationship, parenting skills and parental competencies ${ }^{22,23}$. Positive parenting program's effectiveness has made it highly recommended as a primary intervention ${ }^{29}$. Although many parenting programs are currently available to assist primary caregivers to proactively care for their children and respond to children's behaviours appropriately, such programs are not readily accessible due to limited resources, high costs, and transportation needs to attend face-

to-face parenting programs $\mathbf{s}^{3,20,22,23}$, especially in low-resource settings and perhaps increased difficulties to access such programs during the coronavirus disease 2019 (COVID-19) pandemic. Therefore, many parents have not received this training or, if they have initiated in such training, they often were not able to complete the parent training program ${ }^{3,20,22}$. 
The COVID-19 pandemic which led to lockdown restrictions resulting in family members having to stay at home, study online, and parents working from home have been associated with escalating stress and worsening mental health between parent-child dyads, which subsequently increased the risk of child's behavioural problems and, ultimately, child abuse $\mathrm{e}^{30-32}$. Besides psychological distress, the COVID-19 pandemic significantly intensified parenting-related exhaustion and negative caregivers' perceptions of children's psychological well-being ${ }^{31,32}$. The parenting-related exhaustion was predicted by psychological distress, lower parental resilience, motherhood and fewer perceived social connections ${ }^{32}$. Parental support and perceived control during the pandemic were associated with lower perceived stress and child maltreatment potential ${ }^{30}$. According to a study in Hubei province, China, child behavioural problems during the COVID-19 situation ranged from $4.7-10.3 \%{ }^{33}$. Thus, the findings supported the implementation of preventive parenting programs to support parents and children ${ }^{30,32}$. Online positive parent training programs may offer a suitable way to support parents who deal with their children day-to-day during COVID-19 situation.

Previous studies have shown the effects of online positive parenting programs on parental sense of competence, parental stress, child's health, and behavioural problems, particularly those with more behavioural and emotional disorders. As behavioural and emotional disorders lead to high economic burden, primary prevention for children's behavioural problems should strongly be considered. Evidence regarding such programs as primary preventive measures pre-emptively addressing subclinical behavioural concerns among otherwise healthy caregivers and children is still lacking. Moreover, basic parenting topics as potential targets that have proven to be effective could benefit general practitioners or primary health care providers supporting parents and children in preventing disruptive behaviours and other mental health problems and, therefore, could save the national budget and benefit human capital in the future. To our knowledge, evidence-based, online parent training programs which take into consideration children with low risks for behavioural problems, the pandemic and Thai cultural context are still not available to the Thai public. Therefore, this study aimed to evaluate the effectiveness of the online positive parenting program (Online PPP) designed with cultural consideration and to suit the COVID-19 pandemic at increasing parenting sense of competence. We hypothesised that participants who received the program would report higher levels of parenting competence and positive parenting skills and lower levels of parental stress and children's behavioural problems compared with the control group.

\section{Results}

From 711 interested participants, 314 participants did not meet initial screening inclusion criteria. On further contact of 397 participants via telephone, 211 were excluded due to not meeting eligibility criteria, inability to attend on-site developmental evaluation, or participants declined to participate when more information about the program was shared over the phone. Then, we sent the final screening form via electronic mail, which included the child development and behavioural screening, and parental stress screening forms to interested participants. Of 108 who passed the final screening, 105 attended the in-person developmental evaluation for their child at King Chulalongkorn Memorial Hospital. Finally, 103 participants met all eligibility criteria and were finally randomised into intervention $(n=52)$ and control $(n=51)$ groups (Fig. 1). Detailed demographics were collected at this time as baseline data.

Four participants from the intervention group dropped out of the study. At 8-week follow-up, there were 3 participants from the intervention group, and at 14-week follow-up, there were 2 participants from the intervention group and 1 participant from the control group who did not complete the questionnaire.

\section{Participant Characteristics}

Of 103 caregiver-child dyads randomised (101 [98.1\%] female), 100 main caregivers (97.1\%) were mothers, one participant (0.97\%) was a father, one $(0.97 \%)$ was an uncle, and another one $(0.97 \%)$ main caregiver was a child's cousin. Caregivers' mean age (SD) was approximately 36.3 (4.3) years in the intervention group and 37.2 (3.4) years in the control group. Most parents from both groups had at least a bachelor's degree and had monthly household income of more than 50,000 Baht. The average child age (SD) of intervention and control groups were 54.3 (12.6) and 52.4 (10.7) months, respectively. Other demographic and baseline characteristics of study participants are shown in Table 1. 


\begin{tabular}{|c|c|c|}
\hline \multirow[t]{2}{*}{ Characteristics } & \multicolumn{2}{|l|}{ No. (\%) } \\
\hline & Intervention $(n=52)$ & Control $(n=51)$ \\
\hline \multicolumn{3}{|l|}{ Caregiver's sex } \\
\hline Female & $50(96.2)$ & $51(100.0)$ \\
\hline \multicolumn{3}{|l|}{ Relationship to child } \\
\hline Mother & $49(94.2)$ & $51(100.0)$ \\
\hline Father and other & $3(5.8)$ & $0(0)$ \\
\hline Age, mean (SD), yr & $36.3(4.3)$ & $37.2(3.4)$ \\
\hline \multicolumn{3}{|l|}{ Educational level } \\
\hline Less than bachelor's degree & $3(5.8)$ & $3(5.9)$ \\
\hline At least bachelor's degree & $49(94.2)$ & $48(94.1)$ \\
\hline \multicolumn{3}{|l|}{ Monthly household income (Baht) } \\
\hline$<30,000$ & $1(0.02)$ & $6(0.12)$ \\
\hline $30,000-50,000$ & $10(0.19)$ & $4(0.08)$ \\
\hline $50,000-100,000$ & $18(0.35)$ & $16(0.31)$ \\
\hline$>100,000$ & $23(0.44)$ & $25(0.49)$ \\
\hline \multicolumn{3}{|l|}{ Marital status } \\
\hline Married & $48(0.92)$ & $47(0.92)$ \\
\hline Separated/Divorced & $4(0.08)$ & $4(0.08)$ \\
\hline \multicolumn{3}{|l|}{ Child's Sex } \\
\hline Female & $26(50.0)$ & $27(52.9)$ \\
\hline Child's age, mean (SD), mo & $54.3(12.6)$ & $52.4(10.7)$ \\
\hline \multicolumn{3}{|l|}{ Child's number of siblings } \\
\hline No siblings & $31(59.6)$ & $27(52.9)$ \\
\hline 1 sibling & $19(36.5)$ & $23(45.1)$ \\
\hline 2 siblings & $2(3.8)$ & $1(2.0)$ \\
\hline \multicolumn{3}{|l|}{ Baseline raw scores, mean (SD) } \\
\hline PSOC total score ${ }^{a}$ & $80.94(8.67)$ & $79.55(9.84)$ \\
\hline PSI-SF total score ${ }^{b}$ & $65.23(11.59)$ & $66.00(12.56)$ \\
\hline PSS total score ${ }^{c}$ & $34.71(6.68)$ & $34.82(6.75)$ \\
\hline \multicolumn{3}{|l|}{ PSDQ } \\
\hline Authoritative & $4.42(0.30)$ & $4.36(0.43)$ \\
\hline Authoritarian & $1.63(0.31)$ & $1.58(0.28)$ \\
\hline Permissive & $2.18(0.42)$ & $2.29(0.56)$ \\
\hline Positive parenting score ${ }^{d}$ & $0.62(0.69)$ & $0.49(1.06)$ \\
\hline
\end{tabular}


Prosocial

Emotional

Conduct

Total difficulties score $(>18=$ high behavioural problems)

a $P S O C$ the Parenting Sense of Competence Scale total score: high scores=high sense of parenting competence; ${ }^{\text {b } P S I-S F ~}$ Parenting Stress Index - Short Form total score: $\geq 86=$ severe parenting stress; ${ }^{c} P S S$ Parental Stress Scale total score: score > $72=$ high parenting stress; ${ }^{\mathrm{d}}$ Positive parenting score: Authoritative subtracted by authoritarian and permissive scores; $S D$ standard deviation, $P S D Q$ Parenting Styles and Dimensions Questionnaire, SDQ Strengths and Difficulties Questionnaire

\section{Program Attendance}

From 8 sessions of the program, live program attendance was high with the median of 7 sessions per participant. In each session, the number of participants who had joined the live video conference ranged between 33 to 50 persons per session (mean 39.4). Some participants who were not available during the live, interactive sessions were able to access the recorded videos of such sessions. Thus, attendance took into account both those attending live, interactive sessions on-time and those accessing recorded videos through self-study. The median of program attendance which included viewing self-study video recordings was 8 sessions/person.

\section{Primary Outcome}

Scores at baseline, 8- and 14 weeks after intervention of all parents' and children's outcomes are presented in Tables 2 and 3. Although no significant baseline differences were found in the primary outcome variables at both 8 and 14 weeks after intervention by using Paired t-test and ANOVA, total PSOC scores significantly increased in GEE model (Wald=9.63; $p=0.008$ ), indicating that the total PSOC score of intervention group significantly improved compared to control group over time. Notably, PSOC skill and knowledge subscale score almost demonstrated statistically significant improvement over time (time variable, Wald $=5.99 ; p=0.05$ ); however, this subscale did not significantly differ between groups (group variable) and when considering group by time interaction (group X time in the GEE model). At 8 weeks, all PSOC subscale scores had decreased in the intervention group but had increased in the control group but not statistically significant. While PSOC skill/knowledge subscale and total scores at 14 weeks had an increasing trend in the intervention group more than the control group, albeit not statistically significant difference within and between groups as shown in Table 2. Other subscales of PSOC did not significantly improve (Tables 2 and 3). 
Table 2

Mean Differences of Parents' and Children's Outcomes at 8 and 14 Weeks Within and Between Groups

\begin{tabular}{|c|c|c|c|c|c|c|c|c|c|}
\hline \multirow[t]{4}{*}{ Variables } & \multicolumn{4}{|c|}{ Intervention $(n=52)$} & \multicolumn{4}{|c|}{ Control $(n=51)$} & \multirow{3}{*}{$\begin{array}{l}\text { Change from } \\
\text { baseline between } \\
\text { groups }\end{array}$} \\
\hline & \multirow{2}{*}{\multicolumn{2}{|c|}{$\begin{array}{l}\text { After intervention } \\
\text { (8 weeks- } \\
\text { Baseline) }\end{array}$}} & \multirow{2}{*}{\multicolumn{2}{|c|}{$\begin{array}{l}\text { Change from } \\
\text { baseline } \\
\text { (14 weeks- } \\
\text { baseline) }\end{array}$}} & \multirow{2}{*}{\multicolumn{2}{|c|}{$\begin{array}{l}\text { After intervention } \\
\text { (8 weeks- } \\
\text { Baseline) }\end{array}$}} & \multirow{2}{*}{\multicolumn{2}{|c|}{$\begin{array}{l}\text { Change from } \\
\text { baseline } \\
\text { (14 weeks- } \\
\text { baseline) }\end{array}$}} & \\
\hline & & & & & & & & & \\
\hline & $\begin{array}{l}\text { Mean } \\
(\mathrm{SD})\end{array}$ & p & $\begin{array}{l}\text { Mean } \\
(\mathrm{SD})\end{array}$ & $\mathbf{p}$ & $\begin{array}{l}\text { Mean } \\
\text { (SD) }\end{array}$ & p & $\begin{array}{l}\text { Mean } \\
\text { (SD) }\end{array}$ & p & $\mathrm{p}$ \\
\hline \multicolumn{10}{|l|}{ PSOC } \\
\hline $\begin{array}{l}\text { Perceived } \\
\text { valuing/comfort }\end{array}$ & $\begin{array}{l}-0.48 \\
(4.76)\end{array}$ & 0.499 & $\begin{array}{l}0.69 \\
(6.52)\end{array}$ & 0.469 & $\begin{array}{l}1.31 \\
(5.80)\end{array}$ & 0.112 & $\begin{array}{l}0.86 \\
(4.97)\end{array}$ & 0.227 & 0.883 \\
\hline \multirow{4}{*}{$\begin{array}{l}\text { Skill/knowledge } \\
\text { Total score }\end{array}$} & $\begin{array}{l}-0.37 \\
(3.44)\end{array}$ & & $\begin{array}{l}0.96 \\
(3.53)\end{array}$ & & $\begin{array}{l}0.63 \\
(3.96)\end{array}$ & & $\begin{array}{l}0.58 \\
(3.57)\end{array}$ & & \\
\hline & $\begin{array}{l}-0.85 \\
(6.19)\end{array}$ & & $\begin{array}{l}1.65 \\
(8.35)\end{array}$ & & $\begin{array}{l}1.94 \\
(6.94)\end{array}$ & & $\begin{array}{l}1.44 \\
(6.27)\end{array}$ & & \\
\hline & & 0.470 & & 0.066 & & 0.263 & & 0.256 & 0.599 \\
\hline & & 0.358 & & 0.178 & & 0.051 & & 0.111 & 0.890 \\
\hline PSI & $\begin{array}{l}1.85 \\
(11.12)\end{array}$ & 0.266 & $\begin{array}{l}2.37 \\
(14.04)\end{array}$ & 0.244 & $\begin{array}{l}0.33 \\
(9.37)\end{array}$ & 0.801 & $\begin{array}{l}0.98 \\
(11.02)\end{array}$ & 0.532 & 0.585 \\
\hline PSS & $\begin{array}{l}1.00 \\
(6.40)\end{array}$ & 0.295 & $\begin{array}{l}0.46 \\
(7.24)\end{array}$ & 0.663 & $\begin{array}{l}1.31 \\
(6.37)\end{array}$ & 0.147 & $\begin{array}{l}0.04 \\
(6.32)\end{array}$ & 0.965 & 0.761 \\
\hline \multicolumn{10}{|l|}{ PSDQ } \\
\hline Authoritative & $\begin{array}{l}0.04 \\
(0.37)\end{array}$ & 0.445 & $\begin{array}{l}0.16 \\
(0.37)\end{array}$ & $0.005^{\star}$ & $\begin{array}{l}0.06 \\
(0.34)\end{array}$ & 0.189 & $\begin{array}{l}0.15 \\
(0.42)\end{array}$ & $0.017^{\star}$ & 0.853 \\
\hline Authoritarian & & 0.964 & & 0.967 & & 0.966 & & $>0.999$ & 0.974 \\
\hline Permissive & $\begin{array}{l}0.00 \\
(0.27)\end{array}$ & 0.667 & $\begin{array}{l}0.00 \\
(0.29)\end{array}$ & 0.724 & $\begin{array}{l}-0.00 \\
(0.28)\end{array}$ & $>0.999$ & $\begin{array}{l}0.00 \\
(0.24)\end{array}$ & $0.020^{\star}$ & 0.173 \\
\hline \multirow[t]{2}{*}{$\begin{array}{l}\text { Positive parenting } \\
\text { score }\end{array}$} & $\begin{array}{l}-0.03 \\
(0.48)\end{array}$ & 0.533 & $\begin{array}{l}-0.03 \\
(0.49)\end{array}$ & 0.129 & $\begin{array}{l}0.00 \\
(0.49)\end{array}$ & 0.573 & $\begin{array}{l}-0.16 \\
(0.47)\end{array}$ & $0.018^{*}$ & 0.485 \\
\hline & $\begin{array}{l}0.07 \\
(0.76)\end{array}$ & & $\begin{array}{l}0.18 \\
(0.82)\end{array}$ & & $\begin{array}{l}0.07 \\
(0.83)\end{array}$ & & $\begin{array}{l}0.31 \\
(0.88)\end{array}$ & & \\
\hline
\end{tabular}

${ }^{*} p<0.05$

PSOC Parenting Sense of Competence, PSI Parenting Stress Index, PSS Parental Stress Scale, PSDQ Parenting Styles and Dimensions Questionnaire, SDQ Strengths and Difficulties Questionnaire 


\begin{tabular}{|c|c|c|c|c|c|c|c|c|c|}
\hline \multirow[t]{3}{*}{ Variables } & \multicolumn{4}{|c|}{ Intervention ( $n=52)$} & \multicolumn{4}{|c|}{ Control $(n=51)$} & \multirow{2}{*}{$\begin{array}{l}\text { Change from } \\
\text { baseline between } \\
\text { groups }\end{array}$} \\
\hline & \multicolumn{2}{|c|}{$\begin{array}{l}\text { After intervention } \\
\text { (8 weeks- } \\
\text { Baseline) }\end{array}$} & \multicolumn{2}{|c|}{$\begin{array}{l}\text { Change from } \\
\text { baseline } \\
\text { (14 weeks- } \\
\text { baseline) }\end{array}$} & \multicolumn{2}{|c|}{$\begin{array}{l}\text { After intervention } \\
\text { ( } 8 \text { weeks- } \\
\text { Baseline) }\end{array}$} & \multicolumn{2}{|c|}{$\begin{array}{l}\text { Change from } \\
\text { baseline } \\
\text { (14 weeks- } \\
\text { baseline) }\end{array}$} & \\
\hline & $\begin{array}{l}\text { Mean } \\
\text { (SD) }\end{array}$ & $\mathrm{p}$ & $\begin{array}{l}\text { Mean } \\
\text { (SD) }\end{array}$ & $\mathbf{p}$ & $\begin{array}{l}\text { Mean } \\
\text { (SD) }\end{array}$ & p & $\begin{array}{l}\text { Mean } \\
\text { (SD) }\end{array}$ & $\mathrm{p}$ & p \\
\hline \multicolumn{10}{|l|}{ SDQ } \\
\hline \multirow{3}{*}{$\begin{array}{l}\text { Prosocial } \\
\text { Emotional } \\
\text { Conduct }\end{array}$} & $\begin{array}{l}0.11 \\
(1.39)\end{array}$ & \multirow{2}{*}{$\begin{array}{l}0.597 \\
0.280\end{array}$} & $\begin{array}{l}0.21 \\
(1.41)\end{array}$ & \multirow{2}{*}{$\begin{array}{l}0.312 \\
0.033^{*}\end{array}$} & $\begin{array}{l}-0.02 \\
(1.67)\end{array}$ & \multirow{2}{*}{$\begin{array}{l}0.933 \\
0.062\end{array}$} & $\begin{array}{l}0.18 \\
(1.55)\end{array}$ & 0.415 & \multirow{2}{*}{$\begin{array}{l}0.925 \\
0.578\end{array}$} \\
\hline & \multirow{2}{*}{$\begin{array}{l}-0.22 \\
(1.35)\end{array}$} & & -0.44 & & \multirow{2}{*}{$\begin{array}{l}-0.31 \\
(1.18)\end{array}$} & & \multirow{2}{*}{$\begin{array}{l}-0.30 \\
(1.04)\end{array}$} & $0.046^{*}$ & \\
\hline & & 0.543 & $(1.38)$ & 0.903 & & 0.659 & & 0.322 & 0.440 \\
\hline Attention & $\begin{array}{l}0.09 \\
(0.96)\end{array}$ & $>0.999$ & $\begin{array}{l}0.02 \\
(118)\end{array}$ & 0.799 & $\begin{array}{l}0.06 \\
(0.95)\end{array}$ & 0.924 & $\begin{array}{l}-0.16 \\
(1.13)\end{array}$ & $0.024^{\star}$ & 0.122 \\
\hline \multirow[t]{3}{*}{ Friendship } & $\begin{array}{l}0.00 \\
(1.49)\end{array}$ & \multirow[t]{3}{*}{0.730} & $\begin{array}{l}-0.06 \\
(1.69)\end{array}$ & \multirow[t]{3}{*}{0.346} & $\begin{array}{l}-0.02 \\
(1.46)\end{array}$ & 0.523 & $\begin{array}{l}0.40 \\
(1.21)\end{array}$ & \multirow[t]{3}{*}{0.863} & \multirow[t]{3}{*}{0.598} \\
\hline & $\begin{array}{l}-0.07 \\
(1.27)\end{array}$ & & $\begin{array}{l}-0.21 \\
(1.52)\end{array}$ & & $\begin{array}{l}0.14 \\
(1.52)\end{array}$ & & $\begin{array}{l}-0.04 \\
(1.63)\end{array}$ & & \\
\hline & $\begin{array}{l}-0.20 \\
(3.32)\end{array}$ & & $\begin{array}{l}-0.69 \\
(4.17)\end{array}$ & & $\begin{array}{l}-0.14 \\
(3.23)\end{array}$ & & $\begin{array}{l}-0.10 \\
(3.32)\end{array}$ & & \\
\hline $\begin{array}{l}\text { Total difficulties } \\
\text { score }\end{array}$ & & 0.692 & & 0.259 & & 0.763 & & 0.832 & 0.442 \\
\hline \multicolumn{10}{|l|}{$\star p<0.05$} \\
\hline
\end{tabular}


Table 3

Parents' and Children's Outcomes on Generalised Estimating Equation (GEE) Analysis

\begin{tabular}{|c|c|c|c|c|c|c|c|c|c|c|c|}
\hline \multicolumn{2}{|c|}{ Variables } & \multirow[t]{2}{*}{ Time } & \multicolumn{2}{|c|}{ Mean values (SE) } & \multicolumn{3}{|c|}{ Group } & \multicolumn{2}{|l|}{ Time } & \multicolumn{2}{|c|}{ Group X Time } \\
\hline & & & Intervention & Control & & Wald & $p$ & Wald & p & Wald & $p$ \\
\hline \multirow[t]{11}{*}{ PSOC } & \multirow[t]{3}{*}{$\begin{array}{l}\text { Perceived } \\
\text { valuing/comfort }\end{array}$} & \multirow{3}{*}{$\begin{array}{l}\text { Baseline } \\
8 \text { weeks } \\
14 \\
\text { weeks }\end{array}$} & $\begin{array}{l}40.97 \\
(26.36)\end{array}$ & $\begin{array}{l}40.42 \\
(26.47)\end{array}$ & \multirow[t]{3}{*}{0.02} & & \multirow[t]{3}{*}{0.888} & \multirow[t]{3}{*}{2.27} & \multirow[t]{3}{*}{0.322} & \multirow[t]{3}{*}{4.25} & \multirow[t]{3}{*}{0.119} \\
\hline & & & $\begin{array}{l}40.69 \\
(26.26)\end{array}$ & $\begin{array}{l}41.80 \\
(26.49)\end{array}$ & & & & & & & \\
\hline & & & $\begin{array}{l}41.69 \\
(26.29)\end{array}$ & $\begin{array}{l}41.28 \\
(26.54)\end{array}$ & & & & & & & \\
\hline & \multirow[t]{5}{*}{ Skill/knowledge } & \multirow[t]{3}{*}{ Baseline } & $\begin{array}{l}39.69 \\
(21.91)\end{array}$ & $\begin{array}{l}38.95 \\
(21.86)\end{array}$ & \multirow[t]{5}{*}{0.97} & & \multirow[t]{5}{*}{0.326} & \multirow[t]{5}{*}{5.99} & \multirow[t]{5}{*}{0.050} & \multirow[t]{5}{*}{5.35} & \multirow[t]{5}{*}{0.069} \\
\hline & & & $\begin{array}{l}39.41 \\
(21.88)\end{array}$ & $\begin{array}{l}39.65 \\
(21.80)\end{array}$ & & & & & & & \\
\hline & & & $\begin{array}{l}40.71 \\
(21.90)\end{array}$ & $\begin{array}{l}39.53 \\
(21.85)\end{array}$ & & & & & & & \\
\hline & & 8 weeks & & & & & & & & & \\
\hline & & $\begin{array}{l}14 \\
\text { weeks }\end{array}$ & & & & & & & & & \\
\hline & \multirow[t]{3}{*}{ Total score } & \multirow{3}{*}{$\begin{array}{l}\text { Baseline } \\
8 \text { weeks } \\
14 \\
\text { weeks }\end{array}$} & $\begin{array}{l}80.65 \\
(45.32)\end{array}$ & $\begin{array}{l}79.38 \\
(45.40)\end{array}$ & \multirow[t]{3}{*}{0.13} & & \multirow[t]{3}{*}{0.716} & \multirow[t]{3}{*}{5.01} & \multirow[t]{3}{*}{0.082} & \multirow[t]{3}{*}{9.63} & \multirow[t]{3}{*}{$0.008^{*}$} \\
\hline & & & $\begin{array}{l}80.06 \\
(45.27)\end{array}$ & $\begin{array}{l}81.46 \\
(45.33)\end{array}$ & & & & & & & \\
\hline & & & $\begin{array}{l}82.40 \\
(45.23)\end{array}$ & $\begin{array}{l}80.82 \\
(45.44)\end{array}$ & & & & & & & \\
\hline \multirow[t]{8}{*}{ PSDQ } & \multirow[t]{4}{*}{ Authoritative } & Baseline & $4.40(1.70)$ & 4.37 & 0.40 & & 0.525 & 15.52 & $<0.001^{\star}$ & 0.20 & 0.907 \\
\hline & & 8 weeks & $4.44(1.70)$ & & & & & & & & \\
\hline & & 14 & $4.56(1.70)$ & $\begin{array}{l}4.43 \\
(1.70)\end{array}$ & & & & & & & \\
\hline & & & & $\begin{array}{l}4.51 \\
(1.70)\end{array}$ & & & & & & & \\
\hline & Authoritarian & Baseline & $1.63(1.50)$ & $\begin{array}{l}1.59 \\
(150)\end{array}$ & 0.60 & & 0438 & 0.05 & 0.973 & 0.15 & 0.928 \\
\hline & & 8 weeks & $1.64(1.49)$ & & & & & & & & \\
\hline & & $\begin{array}{l}14 \\
\text { weeks }\end{array}$ & $1.63(1.50)$ & $(1.49)$ & & & & & & & \\
\hline & & & & $\begin{array}{l}1.59 \\
(1.49)\end{array}$ & & & & & & & \\
\hline
\end{tabular}

${ }^{*} p<0.05 ; S E$ standard error

GEE Analysis was based on repeated measures taking into consideration the interaction of group by time.

PSOC Parenting Sense of Competence, $P S D Q$ Parenting Styles and Dimensions Questionnaire, SDQ Strengths and Difficulties Questionnaire 


\begin{tabular}{|c|c|c|c|c|c|c|c|c|c|c|}
\hline \multicolumn{2}{|c|}{ Variables } & \multirow[t]{2}{*}{ Time } & \multicolumn{2}{|c|}{ Mean values (SE) } & \multicolumn{2}{|l|}{ Group } & \multicolumn{2}{|l|}{ Time } & \multicolumn{2}{|c|}{ Group X Time } \\
\hline & & & Intervention & Control & Wald & $p$ & Wald & $\mathrm{p}$ & Wald & p \\
\hline & \multirow[t]{4}{*}{ Permissive } & Baseline & $2.17(3.10)$ & \multirow{2}{*}{$\begin{array}{l}2.32 \\
(3.11)\end{array}$} & \multirow[t]{4}{*}{1.19} & \multirow[t]{4}{*}{0.276} & \multirow[t]{4}{*}{7.10} & \multirow[t]{4}{*}{$0.029 *$} & \multirow[t]{4}{*}{3.12} & \multirow[t]{4}{*}{0.210} \\
\hline & & 8 weeks & $2.15(3.10)$ & & & & & & & \\
\hline & & \multirow{2}{*}{$\begin{array}{l}14 \\
\text { weeks }\end{array}$} & $2.15(3.10)$ & $\begin{array}{l}2.32 \\
(3.11)\end{array}$ & & & & & & \\
\hline & & & & $\begin{array}{l}2.16 \\
(3.09)\end{array}$ & & & & & & \\
\hline & \multirow{4}{*}{$\begin{array}{l}\text { Positive } \\
\text { parenting score }\end{array}$} & Baseline & $0.60(4.74)$ & \multirow{2}{*}{$\begin{array}{l}0.46 \\
(4.74)\end{array}$} & \multirow[t]{4}{*}{0.06} & \multirow[t]{4}{*}{0.805} & \multirow[t]{4}{*}{3.44} & \multirow[t]{4}{*}{0.179} & \multirow[t]{4}{*}{1.26} & \multirow[t]{4}{*}{0.532} \\
\hline & & 8 weeks & $0.66(4.72)$ & & & & & & & \\
\hline & & \multirow{2}{*}{$\begin{array}{l}14 \\
\text { weeks }\end{array}$} & \multirow[t]{2}{*}{$0.78(4.72)$} & $\begin{array}{l}0.53 \\
(4.73)\end{array}$ & & & & & & \\
\hline & & & & $\begin{array}{l}0.77 \\
(4.71)\end{array}$ & & & & & & \\
\hline \multirow[t]{4}{*}{ SDQ } & \multirow[t]{4}{*}{$\begin{array}{l}\text { Total difficulties } \\
\text { score }\end{array}$} & \multirow{4}{*}{$\begin{array}{l}\text { Baseline } \\
8 \text { weeks } \\
14 \\
\text { weeks }\end{array}$} & $\begin{array}{l}8.54 \\
(52.19)\end{array}$ & $\begin{array}{l}8.62 \\
(52.23)\end{array}$ & \multirow[t]{4}{*}{0.04} & \multirow[t]{4}{*}{0.845} & \multirow[t]{4}{*}{7.07} & \multirow[t]{4}{*}{$0.029 *$} & \multirow[t]{4}{*}{5.31} & 0.07 \\
\hline & & & 8.45 & 8.48 & & & & & & \\
\hline & & & (52.12) & $(52.21)$ & & & & & & \\
\hline & & & $\begin{array}{l}7.67 \\
(52.08)\end{array}$ & $\begin{array}{l}8.52 \\
(52.18)\end{array}$ & & & & & & \\
\hline$* p<c$ & SE standard erro & & & & & & & & & \\
\hline GEE & ysis was based o & epeated $\mathrm{n}$ & easures takin & into consi & leration the in & teractic & roup by & time. & & \\
\hline $\begin{array}{l}\text { PSOC } \\
\text { Ques }\end{array}$ & $\begin{array}{l}\text { renting Sense of } \\
\text { naire }\end{array}$ & petence, & $S D Q$ Parer & Styles & Dimensi & ques & & ength & & \\
\hline
\end{tabular}

\section{Secondary Outcomes}

Parental outcomes: PSI-SF, PSS and PSDQ

Parenting stress had a slight increasing trend in both intervention and control groups over time, although not statistically significant in Paired t-test, ANOVA and GEE analysis (Table 2 and Supplementary Table 2). Although permissive subscale and positive parenting score on the PSDQ at 14 weeks demonstrated significant changes from baseline in the control group compared with the intervention group by using Paired t-test, ANOVA analysis showed no significant difference in mean changes between groups in all PSDQ subscales (Table 2). PSDQ authoritative subscale from intervention group clearly improved (mean change $=0.16, S D=0.37 ; p=0.005$ ) at 14 weeks after intervention (Table 2). Supplementary Table 4 had further details of the analysis of components in authoritative and authoritarian subscales on the PSDQ. For GEE analysis (Table 3), we found that PSDQ subscales such as authoritative and permissive parenting styles showed significant score improvement towards more desirable parenting styles within each group over time (time variable: Wald of authoritative $=15.52 ; p<0.001$, Wald of permissive $=7.10 ; p=0.029$ ) but did not significantly differ between groups (group variable) and with group by time interaction (group X time). More detail of components in authoritative and authoritarian subscales on the PSDQ from GEE model are shown in Supplementary Table 2.

\section{Children's outcomes}

Within groups, mean differences of scores at 8 and 14 weeks compared to baseline showed that there were no significant changes in child's behaviours at 8 weeks. At 14 weeks compared with baseline, there was a significant improvement in children's emotional problems in both groups, with a mean difference of $0.44(p=0.033)$ in the intervention group and $0.30(p=0.046)$ in the control group. Moreover, children from the control group were found to have more attention problems (mean difference $0.40, p=0.024$ ). There were no significant differences in behavioural problems, as reflected by total difficulties scores, at 14 weeks compared with baseline both 
within and between groups (Table 2). However, by 14 weeks after the intervention, children's behavioural problems overall decreased in both groups (Wald 7.07, $p=0.029$ ) (Table 3). More detail of SDQ subscales in GEE model are illustrated in Supplementary Table 3.

\section{Discussion}

In this randomised controlled clinical trial of an online positive parenting training program as a potential preventive intervention for previously healthy parent-child dyads, Online PPP significantly improved the primary outcome measure-parents' sense of competence compared to active comparator control group over time. Among the range of other secondary outcomes, parents reported increased authoritative and decreased permissive parenting styles within each group during 14 weeks of the follow-up period, which did not differ between groups. Moreover, significantly reduced children's emotional problems and overall behavioural problems, as reflected by total difficulties scores on the SDQ, were also noted at 6 weeks after the end of the intervention period.

The traditional positive parenting programs such as the Triple P-Positive Parenting Program ${ }^{23}$ and the Korean Parent Training Program ${ }^{34}$ had been shown to have a wide range of desirable child, parent and family outcomes in both short- and long-term. For example, the programs reduced emotional and behavioural problems of children, promoted effective parenting, improved parenting satisfaction and efficacy in addition to strengthened parental relationship similar to previous online positive parenting programs. There is evidence to demonstrate that online positive parenting programs improved parental competency and reduced parental mental health problems and also ameliorated children's behavioural and emotional problems ${ }^{3,20,22,35}$. According to a meta-analysis, components of highly effective positive parenting programs included teaching parental self-control, guiding parents to foster problem-solving skills and self-control in their children, promoting child's development through play, and increasing the quality of time spent together ${ }^{22}$. Consistent with previous traditional and online parenting programs, Online PPP for intervention group in this present study focused on building and strengthening parent-child relationship, supporting caregiver's mental health and well-being, promoting effective communication, fostering daily routine and activities schedule, and managing both desirable and undesirable behaviours through live instruction via video conferencing, discussion and exchange of opinions, in addition to role play for some sessions. Small and whole group discussions in our study utilised transformative learning theory. If caregivers were unable to participate in live sessions, they were able to acquire the basic knowledge via an edited video recording. This modality provided the flexibility for parents to access parenting techniques via video recordings even when they may be busy on the specified day of the live sessions. In the active intervention control group, general knowledge which did not overlap with the specified Online PPP curriculum was provided via communication application. Furthermore, Online PPP was suitable for facilitating and supporting parents in dealing with their children during the COVID-19 pandemic.

Due to the nature of online platform and the flexibility of the program, parents could access sessions that they missed via video recordings. This led to the high "attendance" and completion of all sessions (median=8 sessions/person). Attendance of previous parenting programs were often reported as $35-50 \%$ of all lessons, and only $60 \%$ of parents fully attended the program ${ }^{22}$. Furthermore, the attrition rate was about $33 \%{ }^{20}$ as compared to our study's attrition rate of $7.69 \%$ for the intervention group. Consequently, various online parent training programs have been developed to address accessibility concerns such as multi-point videoconferencing ${ }^{36}$, web-based online parent training sessions and telephone-based program ${ }^{3}$, and tailored program utilizing text messages ${ }^{20}$. These online positive parenting programs have high rates of program satisfaction due to saved cost and travel time in addition to effectiveness as rated by the parents ${ }^{3,20,35-37}$. Program attendance rate was higher in online parent training program with up to $74 \%$ of participants who attended all sessions ${ }^{38}$.

In the intervention group, the parenting sense of competency minimally decreased initially at 8 weeks but later greatly improved at 14 weeks. We suspected that this was likely due to the nature of transformative learning strategy which may take some time to change behaviours, skills and attitudes. As the intervention period coincided with the new wave of the COVID-19 outbreak in Thailand leading to lockdown restriction including public orders to work from home and implement online schooling to minimise the spread of infection, parents had to spend more time with their children and inevitably had to manage more children's problematic behaviours. Moreover, some families had increased exposure to economic and social stressors and worsening mental health of parent-child dyads that resulted from stay-at-home orders ${ }^{30-32}$. These external stressors may, in turn, affect parents' ability to apply lessons learnt for further self-improvement. Thus, this may partially explain the attenuated effect of the intervention at 8 weeks and the lack of change in stress level despite improved parents' sense of competence at 14 weeks. This finding differed from a previous 
study which showed decreased parental distress after parenting intervention ${ }^{20}$. In addition, baseline characteristics of parenting styles were rather positive in both groups and therefore may not change significantly in 14 weeks for both groups. As an example, after 14 weeks of intervention, both groups reported more authoritative parenting style, and the control group reported less permissive parenting style, possibly due to relatively high positive parenting skills at baseline and participants' high education and household income which may lend to more support and means to positive child-rearing practices. Although the raw score of parenting sense of competence in the intervention group may appear to increase minimally at 14 weeks compared to the control group, GEE model showed that total score of PSOC in the intervention group was significantly greater compared to the control group over time.

This research found that emotional concerns within each group significantly improved at 14 weeks for both intervention and control groups. Furthermore, over time, children's behavioural concerns decreased in both groups. This may in part be explained by improved parenting sense of competence over time in intervention group and increased positive parenting, defined as more authoritative and less permissive parenting, in both groups at 14 weeks in addition to relatively high levels of positive parenting reported at baseline. The decrease in emotional and behavioural concerns as caregivers became more responsive and competent in their own parenting skills exemplified the transactional model and bidirectionality in parent-child relationships. Positive parenting has been found to be associated with desirable behaviours such as lower screen time in children ${ }^{39}$. Additionally, searching for positive parenting information online these days has never been easier, which may mean that the control group may attain such information from other sources as it would be unethical to restrict the control group from navigating other online resources for parenting support. Moreover, those in the control group still received weekly general parenting education via group communication application for 8 weeks in the forms of text articles or videos which may increase parental awareness and practice in such topics. As a result, the efficacy of the intervention in reducing behavioural concerns did not differ significantly between the intervention and control groups. This study, along with previous work by Sanders and team, found that both online parent training and self-help workbooks were effective in reducing disruptive behaviours ${ }^{23}$.

The lack of change in stress level as evident on PSS and PSI may be due to the fact that Online PPP included intensive, weekly 1.5hour sessions lasting for 8 weeks with a gentle reminder a day before each scheduled session. The program itself may cause more stress for parents as they had to "take time off" from taking care of their children in order to attend live, interactive sessions. From these outcomes, we found that our online positive parenting program benefited both parents and children.

There are some limitations in this study. First, as this research aimed to administer Online PPP as a modality for primary prevention in order to increase parents' sense of competence and minimise overall behavioural concerns, we specifically selected caregivers with low stress and caregivers and children who did not have any chronic physical or mental health illnesses. This may cause selection bias; however, given the aim of testing Online PPP as a primary preventive measure, we decided to define the inclusion criteria as such. Therefore, both primary and secondary outcomes at various time points may not significantly differ. Secondly, the intervention group required direct discussion with the facilitators based on transformative learning theory and some Thai parents might not feel readily comfortable talking and sharing among strangers. As a result, live, interactive sessions may not be suitable for all Thai parents. In addition, due to the virtual, interactive nature of our intervention, completely blinding participants was not possible. Nonetheless, all caregivers were informed that they will receive parent training via one of the two modes, either live video conference or one-way information-sharing via communication application. None of the participants knew whether they were in intervention or control group and the desired outcomes of the study. Participants received their personal identification code which served as blinding for the researchers analysing the outcomes. Thirdly, caregivers from our study had relatively high education, household income and high positive parenting style at baseline. Consequently, this may limit the generalizability of our results. Fourthly, during the study, there was a new wave of COVID-19 outbreak in Thailand which may have led to the lack of apparent reduction of stress levels in parents. Noteworthily, the stress levels did not statistically significantly increase in both groups. Fifthly, children's behavioural problems measured by Strengths and Difficulties Questionnaire were completed by only the main caregiver, which may not be representative of behavioural problems from other perspectives or settings such as classroom teachers. Nevertheless, given that the main caregiver spent the majority of each day with the child during the COVID-19 pandemic, this measurement could serve as reflection of children's overall behaviours. Sixthly, as the participants were followed up to 14 weeks, this precluded us from determining the long-term effectiveness of Online PPP. Finally, even though our study did not include costeffectiveness analysis as our main outcome, the total cost of hosting Online PPP was estimated to be 4,000 Baht (equivalent to $£ 87.00)$ per family for the whole course. In comparison to the long-term, high annual cost of behavioural and mental health

Page $11 / 18$ 
disorders, preventive programs such as Online PPP may lessen the overall economic burden and the national budget may be better spent investing in building parenting skills. The topics included in Supplementary Table 1 have proven to be effective targets for primary care providers to utilise in virtual clinical settings as tools in increasing parental sense of competence and positive parenting skills and ultimately reducing children's emotional and behavioural concerns.

In spite of these limitations, to the best of our knowledge, this research is the first randomised controlled trial examining the effects of online positive parenting program, designed with the integration of transformative learning theory, on healthy parents and children as compared to general parenting education via a communication application alone in Thailand. Our study further showed that parents had higher parenting sense of competency after Online PPP and more positive parenting styles in both groups, which ultimately led to less emotional and overall behavioural concerns at 14 weeks.

\section{Methods}

\section{Study Design}

We designed a randomised clinical trial to evaluate the effectiveness of Online Positive Parenting Program (Online PPP) in healthy caregivers and their 3- to 6-year-old children. After written informed consent were obtained from all primary caregivers, participants were randomised using computer-generated block of 4 allocations, into two parallel groups, intervention versus active comparator control groups, by K.J. Data were obtained from all participants at baseline, 8 weeks (completion of intervention phase), and 14 weeks after the intervention began. All participants were given information about the study and gave consent. This trial was registered on the Thai Clinical Trial Registry (TCTR20201030001 on 30/10/2020), and reported in accordance to the Consolidated Standards of Reporting Trials (CONSORT) Guidelines.

\section{Sample Size Calculation}

We used G*Power version 3.1.9.2 to calculate sample size which initially yielded 86 parent-child dyads in the Online PPP intervention group and the active comparator control group assuming the effect size of 0.15 , alpha 0.05 , power $80 \%$ with $15 \%$ attrition rate.

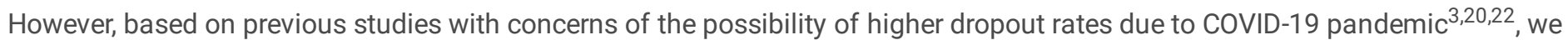
decided to use the target sample size as 103 parent-child dyads to account for $40 \%$ attrition rate.

\section{Selection Criteria}

Targeted research participants were healthy parents and children aged 3-6 years who had no or minimal risk of psychological and behavioural problems. We recruited participants through a social network platform: the Facebook page of our division. All participants received secure online screening form to determine eligibility. Inclusion criteria were as follows: (1) healthy primary caregivers of children aged 3-6 years at the time of recruitment, (2) having low stress levels (Parental Stress Scale $\leq 72^{20}$ and Parent Stress Index - Short Form $<86^{40}$ ) without mental health illnesses, (3) children with birth weight $\geq 2,500$ grams and had no history of pre-, peri- and postnatal complications, (4) healthy children who had normal developmental screening by Developmental Surveillance and Promotion Manual (DSPM) ${ }^{41}$, (5) children with normal behavioural screening by Strengths and Difficulties Questionnaire (SDQ) defined as having total difficulties score $\leq 18^{11}$ and no mental health problems, and (6) the primary caregiver had access to a telephone, computer, and an internet connection in their home.

Participants were excluded if: (1) their child had history of developmental delay or severe behavioural problems, or (2) their child was not able to attend in-person developmental evaluation at King Chulalongkorn Memorial Hospital.

\section{Study procedures}

Eligible participants were randomised into two groups: the Online PPP intervention group and the active comparator control group; separate LINE (Line Corporation, Tokyo, Japan) OpenChat groups were created for each group whereby participants could not directly contact other participants. The participant flowchart (CONSORT diagram) is presented in Figure 1.

At 8 and 14 weeks after the start of the intervention, both groups of participants received a message through a communication application (LINE OpenChat) to complete the follow-up assessments. A reminder message was sent to alert when participants had

Page $12 / 18$ 
not completed the assessments. A follow-up telephone call was made to the participant if the assessment was not completed after 2 reminders. Project participants were given 500 Baht (equivalent to £10.96) per person for participating in the project and completing the assessment at every time point.

\section{Interventions}

Between October to December 2020, participants in both the Online PPP intervention group and the active comparator group received similar weekly general parenting education via group communication application (LINE OpenChat groups) for 8 weeks in the forms of text articles or videos.

In the Online PPP intervention group, intensive video conferencing via Zoom platform (Zoom Video Communications, Inc., San Jose, California, USA) was conducted once a week for 8 weeks, with the scheduled date, time, and link and the reminder for each session's attendance sent via the LINE OpenChat. Each video conference session took 90-120 minutes and utilised transformative learning theory. In some sessions, there were small breakout groups with trained facilitators. The content included empowering parents, shifting the focus back to building a good parent-child relationship, fostering positive and effective communication, mastering behaviour modification techniques, organizing daily life routine, supporting self-care of parents, promoting development through play and storytelling (Supplementary Table 1). Recorded videos of the live session were edited and available for parents who were unable to attend each week's activity. Furthermore, participants in the intervention group were able to ask specific questions about learning contents and their concerns regarding their children's behaviours via Line OpenChat during the intervention period, whereas such concerns raised by participants in the control group were not responded to until study completion at 14 weeks.

\section{Measures}

At baseline, we collected demographic data including parents' sex, age, marital status, and education level. Data on household income, child's age, and sex were also recorded. Primary outcome was parental competence and secondary outcomes were parenting skills, parental stress and child's behavioural problems assessed at baseline, 8 weeks (end of intervention phase), and 14 weeks after the intervention began.

\section{Outcome Measures}

Parenting Sense of Competence

The Parenting Sense of Competence Scale Thai Version (PSOC), a validated questionnaire, consisted of 17 self-report and selfadministered items assessing parent's perception of their own parenting performance ${ }^{16,42}$. To date, the PSOC tool was designed for parents of children aged 0-17 years old ${ }^{42}$. PSOC scale had 2 categories: skill/knowledge 8 items and perceived value/comfort 9 items with the internal consistency for skill/knowledge of 0.73 and perceived value/comfort of 0.80 . Total scores could range between 17-102 points with higher total scores suggesting a higher sense of parenting competence.

Parenting Styles

Parenting Styles and Dimensions Questionnaire-short version (PSDQ-short version): Thai version included 32 self-report items evaluating different parenting styles in each parent including authoritative, authoritarian, and permissive parenting style. The internal consistency of the PSDQ (short version) in 3-year-old children were $0.80,0.75$, and 0.62 for authoritative, authoritarian, and permissive parenting style, respectively ${ }^{39}$. Such variables were relatively comparable to a pioneering work by Robinson et al. which demonstrated the internal consistency of $0.86,0.82$, and 0.64 for authoritative, authoritarian, and permissive parenting style, respectively ${ }^{43}$.

Parental Stress

Parental Stress Scale (PSS) had 18 Likert-scale, self-administered questions regarding the degree of parental stress with the alpha coefficient of 0.83 and test-retest reliability of $0.81^{44}$. Parental Stress Scale, Thai version was divided into 8 positive items and 10 negative items with Cronbach's alpha coefficient of 0.81 and reliability of $0.89^{13}$. Possible scores ranged between 18 to 90 with higher scores indicating higher parenting stress level. 
The Thai version of the Parenting Stress Index - Short Form (PSI-SF) was a self-report, 36-item questionnaire assessing both parenting stress and particularly the stress in child-parent relationship with Cronbach's alpha coefficient of $0.94^{40}$. The questionnaire was divided into 3 areas as follows: (1) Parental Distress (PD): 12 items, (2) Parent-Child Dysfunctional Interaction (P-DI): 12 items, and (3) Difficult Child (DC): 12 items. Responses were based on a 5-point Likert scale with total score higher than the 85th percentile or a raw score $\geq 86$ points representing clinically significant parenting stress level.

\section{Child's Behavioural Concerns}

Strengths and Difficulties Questionnaire (SDQ) was a highly reliable, 25-item, 3-point Likert scale questionnaire assessing behaviours of children aged 4-16 years ${ }^{11}$. Higher scores for conduct problems, hyperactivity, emotional problems, and peer problems, and total difficulties, sum of the 4 aforementioned domains, indicated greater behavioural problems.

\section{Statistical Analyses}

Baseline data from all randomised participants were summarised by treatment group. Continuous variables are presented as means and standard deviations and categorical variables as frequency and percentage. Primary and secondary outcomes were summarised descriptively by treatment group at each time point. The primary and secondary outcomes analyses used the intentionto-treat (ITT) population that included all randomised participants. All study outcomes within groups at both 8 and 14 weeks were analysed using Paired t-test and Analysis of Variance (ANOVA) was performed to analyse outcomes between groups at 14 weeks. For the outcomes that were not normally distributed and measured at various time points, we used Generalised Estimating Equations (GEE) model to estimate the parameters of a generalised linear regression and determine the interaction between 2 groups over 3 time points. We adjusted potential confounding factors including household income, caregiver's age, education, number of child's siblings, child' age and gender. Moreover, we applied natural log transformation to adjust the non-normally distributed data for GEE analysis. GEE was computed with mean values, standard error (SE) and Wald test for each independent variable. Statistical significance was defined as $p$ values $<0.05$. Statistical analyses were performed using SPSS statistical software version 22 (IBM Inc., Bangkok, Thailand) for windows with the support from Chulalongkorn University.

\section{Ethical Consideration}

This study was approved by the Institutional Review Boards of the Faculty of Medicine, Chulalongkorn University (IRB 310/63). All procedures were performed in accordance with the International Council on Harmonisation (ICH) Guidance for Industry, E6 Good Clinical Practice: Consolidated Guidance (ICH-E6) to ensure research integrity and protect human subjects.

\section{Conclusion}

Online positive parenting program formats, either through live, interactive video conferencing or one-way communication application, enhanced the access of parenting training and led to more positive parenting and reduced children's behavioural problems. Online PPP specifically increased parents' sense of competence compared to active comparator control group. However, the intensity and duration of live, interactive video conference training sessions may need to be adjusted to better suit the Thai culture. Online PPP offers an innovative positive parenting training model suited for the "new normal" era limited by social distancing requirements and may provide some guidance on further development of online parent training modules aimed to increase access for parents near and far.

\section{Declarations}

\section{Data Availability}

The datasets generated and analysed during the current study are available from the corresponding author on reasonable request.

\section{Acknowledgements}

We are grateful to all participants and their families who participated in this study; Professor Charlotte Johnston and Asst. Prof. Dr. Tatirat Tachasuksri who had kindly given us permission to use the PSOC and PSOC Thai version; Chuthamas Payjapoh who gave permission to use the PSS Thai version; Asst. Prof. Dr. Nuttorn Pityaratstian for granting permission to use PSI-SF Thai version; 
Department of Mental Health for granting the permission for us to use SDQ Thai version; Dr. Kobrat Chiraphadhanakul, Dr. Pornchada Srisinghasongkram, Dr. Prapasri Nuntnarumit, Dr. Lalin Rungsattatharm, Dr. Priyakorn Tasingha, Montira Sangritdech, Amornrat Sukkul and Sukonta Sinthop who kindly assisted us during the parent training program.

\section{Author Contributions}

S.T. and I.K. were responsible equally for study design, acquisition and interpretation of data, drafting the article, and approved the final version to be published. P.T. had substantial contributions to conception and design, acquisition and interpretation of data, drafted and revised the article critically for important intellectual content, and finally approved the version to be published. K.J. was responsible for data analysis, revised the article critically for important intellectual content; and finally approved the version to be published. W.C.: had substantial contributions to conception and design, acquisition of data, analysis, and interpretation of data; drafted the article and revised it critically for important intellectual content; and finally approved the version to be published.

\section{Competing Interests}

The authors declare no competing interests.

\section{Ethics approval}

This study was approved by the Institutional Review Boards of the Faculty of Medicine, Chulalongkorn University (IRB 310/63).

\section{Financial support}

This work was supported by the grant from Broadcasting and Telecommunications Research and Development Fund for the Public Interest (Grant number A62-1-(2)-002).

\section{References}

1. Licence, K. Promoting and protecting the health of children and young people. Child Care Health Dev. 30, 623-635, doi:https://doi.org/10.1111/j.1365-2214.2004.00473.x (2004).

2. Polanczyk, G. V., Salum, G. A., Sugaya, L. S., Caye, A. \& Rohde, L. A. Annual Research Review: A meta-analysis of the worldwide prevalence of mental disorders in children and adolescents. J. Child Psychol. Psychiatry 56, 345-365, doi:https://doi.org/10.1111/jcpp.12381 (2015).

3. Sourander, A. et al. Internet-assisted parent training intervention for disruptive behavior in 4-year-old children: a randomized clinical trial. JAMA Psychiatry 73, 378-387, doi:https://doi.org/10.1001/jamapsychiatry.2015.3411 (2016).

4. Burke, J. D., Loeber, R. \& Birmaher, B. Oppositional defiant disorder and conduct disorder: a review of the past 10 years, part II. J. Am. Acad. Child Adolesc. Psychiatry 41, 1275-1293, doi:https://doi.org/10.1097/00004583-200211000-00009 (2002).

5. Achenbach, T. M., Howell, C. T., McConaughy, S. H. \& Stanger, C. Six-year predictors of problems in a national sample: III. Transitions to young adult syndromes. J. Am. Acad. Child Adolesc. Psychiatry 34, 658-669, doi:https://doi.org/10.1097/00004583-199505000-00018 (1995).

6. Caspi, A., Moffitt, T. E., Newman, D. L. \& Silva, P. A. Behavioral observations at age 3 years predict adult psychiatric disorders: Longitudinal evidence from a birth cohort. Arch. Gen. Psychiatry 53, 1033-1039, doi:https://doi.org/10.1001/archpsyc.1996.01830110071009 (1996).

7. Loeber, R., Burke, J. D., Lahey, B. B., Winters, A. \& Zera, M. Oppositional defiant and conduct disorder: a review of the past 10 years, part I. J. Am. Acad. Child Adolesc. Psychiatry 39, 1468-1484, doi:https://doi.org/10.1097/00004583-200012000-00007 (2000).

8. Kim-Cohen, J. et al. Prior juvenile diagnoses in adults with mental disorder: developmental follow-back of a prospectivelongitudinal cohort. Arch. Gen. Psychiatry 60, 709-717, doi:https://doi.org/10.1001/archpsyc.60.7.709 (2003).

9. Simonoff, E. et al. Predictors of antisocial personality: Continuities from childhood to adult life. Br. J. Psychiatry 184, 118-127, doi:https://doi.org/10.1192/bjp.184.2.118 (2004).

10. Sourander, A. et al. Childhood predictors of male criminality: a prospective population-based follow-up study from age 8 to late adolescence. J. Am. Acad. Child Adolesc. Psychiatry 45, 578-586, doi:https://doi.org/10.1097/01.chi0000205699.58626.b5 
(2006).

11. Woerner, W., Nuanmanee, S., Becker, A., Wongpiromsarn, Y. \& Mongkol, A. Normative data and psychometric properties of the Thai version of the Strengths and Difficulties Questionnaire (SDQ). J. Ment. Health Thai. 19, 42-57, doi:https://doi.org/10.14456/dmhjournal.2010.21 (2011).

12. Sourander, A. et al. Childhood predictors of psychiatric disorders among boys: a prospective community-based follow-up study from age 8 years to early adulthood. J. Am. Acad. Child Adolesc. Psychiatry 44, 756-767, doi:https://doi.org/10.1097/01.chi.0000164878.79986.2f (2005).

13. Klongdee K, Nintachan P \& Sangon S. Factors related to parenting stress in caregivers of children with Attention Deficit Hyperactivity Disorder. J. Psychiatr. Ment. Health Nurs. 30, 52-68 (2016).

14. Khamon A, Charnsil C, Srisurapanont M \& Suradom C. Development and validation of the Thai version of the Alabama Parenting Questionnaire (APQ). J. Ment. Health Thai. 27, 107-120 (2019).

15. Patel, M. J., Patel, P. B. \& Bansal, R. Parental Stress Scale: Translation and preliminary testing for a Gujarati sample. Natl. J. Community Med. 7, 551-554 (2016).

16. Johnston, C. \& Mash, E. J. A measure of parenting satisfaction and efficacy. J. Clin. Child Psychol. 18, 167-175, doi:https://doi.org/10.1207/s15374424jccp1802_8 (1989).

17. Reece, S. M. \& Harkless, G. Self-efficacy, stress, and parental adaptation: Applications to the care of childbearing families. J. Fam. Nurs.4, 198-215, doi:https://doi.org/10.1177\%2F107484079800400206 (1998).

18. Shonkoff, J. P. et al. The lifelong effects of early childhood adversity and toxic stress. Pediatrics 129, e232-e246, doi:https://doi.org/10.1542/peds.2011-2663 (2012).

19. Gilmore, L. \& Cuskelly, M. Factor structure of the parenting sense of competence scale using a normative sample. Child Care Health Dev. 35, 48-55, doi:https://doi.org/10.1111/j.1365-2214.2008.00867.x (2009).

20. Chu, J. T. W. et al. Effect of MyTeen sms-based mobile intervention for parents of adolescents: A randomized clinical trial. JAMA Netw. Open 2, e1911120-e1911120, doi:https://doi.org/10.1001/jamanetworkopen.2019.11120 (2019).

21. Rajan, S., Jahromi, L., Bravo, D., Umaña-Taylor, A. \& Updegraff, K. Maternal Self-Efficacy Is Protective for Child (but Not Mother) Body Mass Index Among Mexican-Origin Children with Negative Temperament. J. Dev. Behav. Pediatr. 40, 633-641, doi:10.1097/dbp.0000000000000696 (2019).

22. Thongseiratch, T., Leijten, P. \& Melendez-Torres, G. Online parent programs for children's behavioral problems: a meta-analytic review. Eur. Child Adolesc. Psychiatry, 1-14, doi:https://doi.org/10.1007/s00787-020-01472-0 (2020).

23. Sanders, M. R., Kirby, J. N., Tellegen, C. L. \& Day, J. J. The Triple P-Positive Parenting Program: a systematic review and metaanalysis of a multi-level system of parenting support. Clin. Psychol. Rev. 34, 337-357, doi:https://doi.org/10.1016/j.cpr.2014.04.003 (2014).

24. Dretzke, J. et al. The effectiveness and cost-effectiveness of parent training/education programmes for the treatment of conduct disorder, including oppositional defiant disorder, in children. Health. Technol. Assess. 9, iii, ix-x, 1, doi:https://doi.org/10.3310/hta9500 (2005).

25. Lundahl, B., Risser, H. J. \& Lovejoy, M. C. A meta-analysis of parent training: Moderators and follow-up effects. Clin. Psychol. Rev. 26, 86-104, doi:https://doi.org/10.1016/j.cpr.2005.07.004 (2006).

26. Waddell, C., Hua, J. M., Garland, O. M., Peters, R. D. \& McEwan, K. Preventing mental disorders in children: a systematic review to inform policy-making. Can. J. Public Health 98, 166-173, doi:https://doi.org/10.1007/bf03403706 (2007).

27. Dretzke, J. et al. The clinical effectiveness of different parenting programmes for children with conduct problems: a systematic review of randomised controlled trials. Child Adolesc. Psychiatry Ment. Health 3, 1-10, doi:https://doi.org/10.1186/1753-2000-37 (2009).

28. Kazdin, A. E. Bridging the enormous gaps of theory with therapy research and practice. J. Clin. Child Adolesc. Psychol. 30, 59-66, doi:https://doi.org/10.1207/s15374424jccp3001_7 (2001).

29. Pilling, S., Gould, N., Whittington, C., Taylor, C. \& Scott, S. Recognition, intervention, and management of antisocial behaviour and conduct disorders in children and young people: summary of NICE-SCIE guidance. BMJ 346, doi:https://doi.org/10.1136/bmj.f1298 (2013). 
30. Brown, S. M., Doom, J. R., Lechuga-Peña, S., Watamura, S. E. \& Koppels, T. Stress and parenting during the global COVID-19 pandemic. Child Abuse Negl. 110, 104699, doi:https://doi.org/10.1016/j.chiabu.2020.104699 (2020).

31. Raviv, T. et al. Caregiver Perceptions of Children's Psychological Well-being During the COVID-19 Pandemic. JAMA Netw. Open 4, e2111103-e2111103, doi:https://doi.org/10.1001/jamanetworkopen.2021.11103 (2021).

32. Marchetti, D. et al. Parenting-related exhaustion during the italian COVID-19 lockdown. J. Pediatr. Psychol. 45, 1114-1123, doi:https://doi.org/10.1093/jpepsy/jsaa093 (2020).

33. Liu, Q. et al. The prevalence of behavioral problems among school-aged children in home quarantine during the COVID-19 pandemic in china. J. Affect Disord. 279, 412-416, doi:https://doi.org/10.1016/j.jad.2020.10.008 (2021).

34. Kim, E. et al. Pilot Study of the Korean Parent Training Program Using a Partial Group-Randomized Experimental Study. J. Child Adolesc. Psychiatr. Nurs. 27, 121-131 (2014).

35. Moon, R. Y., Mathews, A., Oden, R. \& Carlin, R. Mothers' Perceptions of the Internet and Social Media as Sources of Parenting and Health Information: Qualitative Study. J. Med. Internet Res. 21, e14289, doi:https://doi.org/10.2196/14289 (2019).

36. Shah, R. et al. Participating from homes and offices: Proof-of-concept study of multi-point videoconferencing to deliver group parent training intervention for attention-deficit/ hyperactivity disorder. Asian J. Psychiatr. 41, 20-22, doi:https://doi.org/10.1016/j.ajp.2019.03.006 (2019).

37. Gladstone, T. et al. Effect of internet-based cognitive behavioral humanistic and interpersonal training vs internet-based general health education on adolescent depression in primary care: a randomized clinical trial. JAMA Netw. Open 1, pii: e184278, doi:https://doi.org/10.1016/j.ajp.2019.03.006 (2018).

38. Wetterborg, D. et al. A pilot randomized controlled trial of Internet-delivered parent training for parents of teenagers. J. Fam. Psychol., doi:https://doi.org/10.1037/fam0000541 (2019).

39. Detnakarintra, K., Trairatvorakul, P., Pruksananonda, C. \& Chonchaiya, W. Positive mother-child interactions and parenting styles were associated with lower screen time in early childhood. Acta Paediatr. (Oslo, Norway : 1992) 109, 817-826, doi:https://doi.org/10.1111/apa.15007 (2020).

40. Dangnumkoo, D. Parenting stress in caretakers of children with ADHD, Chulalongkorn University, (2006).

41. Sirithongthaworn, S. The Development of Developmental Surveillance and Promotion Manual; DSPM. J. Psychiatr. Assoc. Thailand 63, 3-12 (2018).

42. Suwansujarid, T., Vatanasomboon, P., Gaylord, N. \& Lapvongwatana, P. Validation of the parenting sense of competence scale in fathers: Thai version. Southeast Asian J. Trop. Med. Public Health 44, 916 (2013).

43. Robinson, C. C., Mandleco, B., Olsen, S. F. \& Hart, C. H. in Handbook of family measurement techniques: Vol 3 Instruments \& index 3 (eds B F Perlmutter, J Touliatos, \& G W Holden) 319-321 (Sage, 2001).

44. Berry, J. O. \& Jones, W. H. The parental stress scale: Initial psychometric evidence. J. Soc. Pers. Relat. 12, 463-472, doi:https://doi.org/10.1177/0265407595123009 (1995).

\section{Figures}


711 Screened

314 Did not meet screening inclusion criteria

211 Did not meet inclusion criteria or declined to participate after informed by telephone

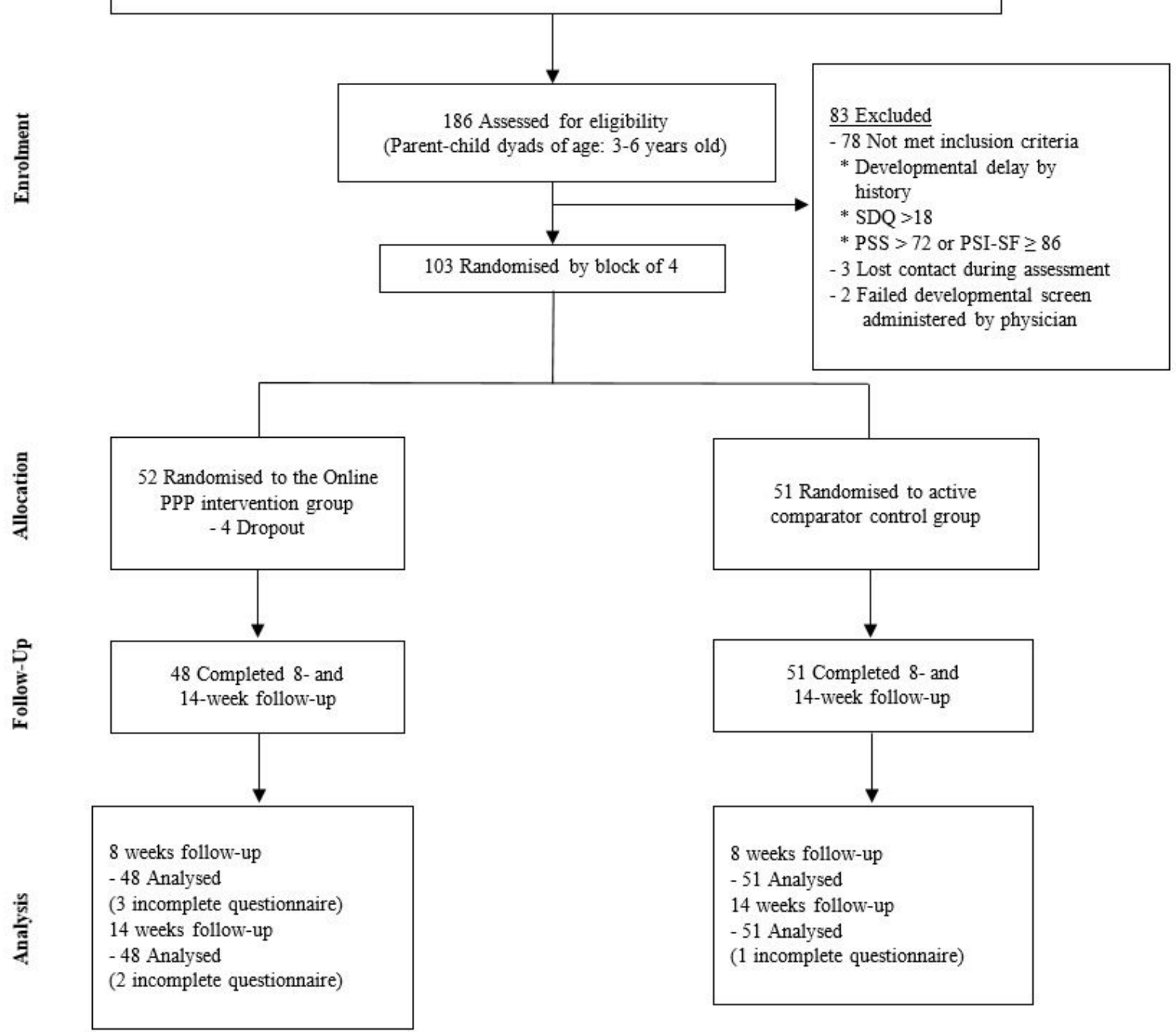

Figure 1

Participant Flow Diagram of Randomised Controlled Trial with Intention-to-Treat Analysis

\section{Supplementary Files}

This is a list of supplementary files associated with this preprint. Click to download.

- SupplementaryMaterial.docx 